\title{
The impact of realistic models of mass segregation on the event rate of extreme-mass ratio inspirals and cusp re-growth
}

\author{
Pau Amaro-Seoane ${ }^{1,2}$ and Miguel Preto ${ }^{3}$ \\ ${ }^{1}$ Max Planck Institute for Gravitational Physics \\ (Albert Einstein Institute), Potsdam-Golm, Germany \\ ${ }^{2}$ Institut de Ciències de l'Espai (CSIC-IEEC) \\ Campus UAB, Torre C-5, parells, ${ }^{\text {na }}$ planta, ES-08193, Bellaterra, Spain \\ ${ }^{3}$ Astronomisches Rechen-Institut (ZAH) \\ Mönchhofstr. 12-14 D-69120 Heidelberg, Germany \\ E-mail: Pau.Amaro-Seoane@aei.mpg.de,miguelp@ari.uni-heidelberg.de
}

\begin{abstract}
One of the most interesting sources of gravitational waves (GWs) for LISA is the inspiral of compact objects on to a massive black hole (MBH), commonly referred to as an "extreme-mass ratio inspiral" (EMRI). The small object, typically a stellar black hole (bh), emits significant amounts of GW along each orbit in the detector bandwidth. The slowly, adiabatic inspiral of these sources will allow us to map spacetime around MBHs in detail, as well as to test our current conception of gravitation in the strong regime. The event rate of this kind of source has been addressed many times in the literature and the numbers reported fluctuate by orders of magnitude. On the other hand, recent observations of the Galactic center revealed a dearth of giant stars inside the inner parsec relative to the numbers theoretically expected for a fully relaxed stellar cusp. The possibility of unrelaxed nuclei (or, equivalently, with no or only a very shallow cusp, or core) adds substantial uncertainty to the estimates. Having this timely question in mind, we run a significant number of direct-summation $N$-body simulations with up to half a million particles to calibrate a much faster orbitaveraged Fokker-Planck code. We show that, under quite generic initial conditions, the time required for the growth of a relaxed, mass segregated stellar cusp is shorter than a Hubble time for MBHs with $M_{\bullet} \lesssim 5 \times 10^{6} M_{\odot}$ (i.e. nuclei in the range of LISA). We then investigate the regime of strong mass segregation (SMS) for models with two different stellar mass components. Given the most recent stellar mass normalization for the inner parsec of the Galactic center, SMS has the significant impact of boosting the EMRI rates by a factor of $\sim 10$ in comparison to what would result from a $7 / 4-$ Bahcall \& Wolf cusp resulting in $\sim 250$ events per Gyr per Milky Way type galaxy. Such intrinsic rate should translate roughly into $\sim 10^{2}-7 \times 10^{2}$ sbh's EMRIs detected by LISA over a mission lifetime of 2 or 5 years, respectively), depending on the detailed assumptions regarding LISA detection capabilities.
\end{abstract}




\section{Introduction}

Nowadays it is well-established that a massive dark object, very possibly a massive black hole $(\mathrm{MBH})$ with a mass of about $4 \times 10^{6} M_{\odot}$, is lurking in the centre of the Milky Way (Eisenhauer et al. 2005; Ghez et al. 2005, 2008; Gillessen et al. 2009). While there is an emerging consensus about the origin and growth of supermassive black holes (SMBH, with masses about or larger than $10^{8} M_{\odot}$ ) (Ferrarese \& Ford 2005; DiMatteo et al. 2008; Volonteri 2010), MBHs with smaller masses such as the one in the Galactic centre remain a (relatively) understudied enigma. One of the keys to understanding the growth and evolution of MBHs in this lower mass range resides in the dynamics of stars in their vicinity. This is the case mainly because relaxation times there are low enough that the surrounding stellar systems should have had enough time - through two-body relaxation alone - to evolve towards a steady-state which is independent of the particular initial conditions at the time of formation. The Galactic center is thought to fulfill such condition. It is the universality of such relaxed stellar nuclei that gives us a crucial predictive power on the expected properties of the $\mathrm{MBH}$ environment, on the stellar candidates for close interaction with the central $\mathrm{MBH}$ and on the resulting gravitational wave $(\mathrm{GW})$ signatures. If, on the contrary, non-relaxed systems were generic, then one would need to resort to case-by-case modelling of each galactic nucleus.

The ideal probe for these innermost regions of galaxies is the GW radiation that is emitted by stellar bhs and other compact objects that come very close to the MBH. One of the main channels for interaction between stars and a central MBH is the adiabatic, slow inspiral of compact remnants (CR) into the $\mathrm{MBH}$ due to the emission of GWs - an EMRI. During such an event, the small body effectively acts as a probe of spacetime close to the $\mathrm{MBH}$ as its orbit slowly shrinks due to the energy and angular momentum lost in the form of GW radiation. In case of $10^{5}-10^{6} M_{\odot}$ MBHs, after some $\sim 10^{4}-10^{5}$ orbits in the LISA band $\left(f_{\text {orb }} \gtrsim 10^{-4} \mathrm{~Hz}\right.$ and a periapsis $a \lesssim$ few $\times \mathrm{R}_{\text {Schw }}$, since we only consider sources which are completely embedded in the band, and not bursting sources), the small body eventually merges with the MBH. The information contained in the waves will allow us to determine the parameters of these binary system with an unprecedented accuracy (see for instance Barack and Cutler 2004; Babak et al. 2010), corroborate the existence of $\mathrm{MBHs}$ and maybe even provide the first direct detection of an intermediate $\mathrm{MBH}$ (in case the primary is $\sim 10^{3-4} M_{\odot}$ ).

$L I S A$ will thus scrutinize exactly the mass range about which electromagnetic observational information is currently lacking. In its most general form, the EMRI problem - the astrophysical modelling of event rates and parameters for EMRIsspans many orders of magnitude. From the bulge regions at few $\times 10 \mathrm{pc}$, where the dynamics is essentially collisionless -but from where single stellar bhs and binaries with CRs originate; down to the parsec scale of the nucleus itself which evolves secularly over (local) relaxation timescales; and then further down to milliparsec scales where relativistic effects start to dominate the evolution. But, however, once a steady state configuration establishes itself in the central parsec region, the EMRI rates are rather 
expected to depend strongly on the (universal) density distribution of CRs within (in order of magnitude) $\mathcal{O}(0.01 \mathrm{pc})$ from the hole. This is indeed the region from which these inspiralling sources are expected to originate (Hopman and Alexander 2005). The dynamics in this tiny volume has been rather unexplored until the relevance of EMRIs and sub-parsec observations of the Galactic center have raised its interest. Since then, many authors have devoted a number of works to the analysis of this peculiar regime (Sigurdsson and Rees 1997; Freitag 2003; Alexander and Hopman 2003; Hopman and Alexander 2006).

We discuss in this work the stellar distribution of dense stellar systems around MBHs in the LISA mass range. Realistic modeling of mass segregationwhich is the natural outcome for any realistic stellar population-will strongly impact the expected EMRI rates, since it favors the accumulation of heavier objects towards the center (Hopman and Alexander 2006; Alexander and Hopman 2009; Preto and Amaro-Seoane 2010a). In Section 2, we begin by summarizing the results obtained by Preto and Amaro-Seoane (2010a) that show how to calibrate the FP calculations with direct $N$-body simulationst; then, still in the same section, we present new results concerning the robustness of $N$-body realisations of stellar cusp growth with respect to the total particle number $N$. In section 3, we present new results on the growth of stellar cusps from a variety of initial conditions resulting from carving a cavity in the star's phase space distribution function. This is motivated from a variety of astrophysical mechanisms that may lead to cusp destruction; and these mechanisms are critically assessed in the end of the section. We show that, under very generic circumstances, the time required for the growth of a cusp is shorter than a Hubble time. Therefore, quasi-steady, mass segregated, stellar cusps are expected to be common around MBHs in the LISA mass range. But see Merritt (2010) and Madigan et al. (2010) for different perspectives. EMRI detection rates for $L I S A$ are expected to peak for $M_{\bullet} \sim 10^{5}-10^{6} M_{\odot}$ (Gair 2009) leading us to conclude that at least a sizeable fraction of these events should originate from strongly segregated cusps. Finally, in Section 4 we present new estimates on the expected EMRI rates in mass segregated nuclei and conclude that our realistic modeling of mass segregation has a significant impact on these rates.

\section{Mass segregation}

The distribution of stars around a massive black hole is a classical problem in stellar dynamics (Bahcall and Wolf 1976; Lightman and Shapiro 1977). Bahcall and Wolf (1976) have shown, through a kinetic treatment that, within the radius of gravitational influence of the hole $r_{h}$, in case all stars are of the same mass, this quasi-steady distribution takes the form of power laws, $\rho(r) \sim r^{-\gamma}$, in physical space with $\rho(r)$ the stellar density at a radius $r$ and $f(E) \sim E^{p}$ in energy space (with $E$ the

$\ddagger$ Direct $N$-body simulations compute the gravitational accelerations between particles using the exact Newton's law, without introducing any approximations to compute the gravitational potential (Binney and Tremaine 2008). 
energy and $\gamma=7 / 4$ and $p=\gamma-3 / 2=1 / 4) \delta$. This is the so-called zero-flow solution for which the net flux of stars in energy space is precisely zero. Preto et al. (2004) and Baumgardt et al. (2004) were the first to demonstrate the robustness of the corresponding direct-summation $N$-body realizations, and have therefore validated the assumptions inherent to the Fokker-Planck (FP) approximation-namely, that scattering is dominated by uncorrelated, 2-body encounters and, in particular, dense stellar cusps $\llbracket$ populated with stars of the same mass are robust against ejection of stars from the cusp. The latter result is not trivial as for a BW $\gamma=7 / 4$ cusp stellar densities are extremely high at the center and the fraction of stars with speeds close to the escape velocity from the cusp is quite high at all radii $r \lesssim r_{h}$, with $r_{h}$ the influence radius of the MBH (Preto 2010).

Single mass models are very poor approximations of real stellar populations. To first order of approximation, an evolved stellar population can be represented by two (well-separated) mass scales: one in the range $\mathcal{O}\left(1 M_{\odot}\right)$ corresponding to low mass mainsequence stars, white dwarfs (WDs) and neutron stars (NSs); another with $\mathcal{O}\left(10 M_{\odot}\right)$ representing stellar bhs. Therefore, for simplicity, here we restrict our discussion to models with two mass components and leave the more general case to another work in preparation (Preto and Amaro-Seoane 2010b).

When stars of two different masses are present, there is mass segregation which is a process by which the heavy stars accumulate near the center while the lighter ones float outward (Spitzer 1987; Khalisi et al. 2007). Accordingly, stars with different mass get distributed with different density profiles. Bahcall and Wolf (1977), henceforth BW77, have argued heuristically that a scaling relation $p_{i}=m_{i} / m_{j} \times p_{j}$ (where the subindices $i, j$ refer to the light or heavy components) establishes itself and depends only on the mass ratio. Here, as in the single-mass case, the crucial assumption is that all components are abundant enough that they undergo enough scattering among themselves and with the other components as to stabilize into an approximate zero-flow solution. Obviously, this cannot happen independently of the number fraction of the different stellar masses (Alexander and Hopman 2009; Preto and Amaro-Seoane 2010a). In the realistic situation where the number fraction of heavy objects (in our case, stellar bhs) is small, a new solution coined by Alexander and Hopman (2009) as strong mass segregation (SMS) obtains with density of heavy objects scaling as $\rho_{H}(r) \sim r^{-\alpha}$, where $\alpha \gtrsim 2$. The solution has two branches and can be parametrized by the parameter

$$
\Delta=\frac{D_{H H}^{(1)}+D_{H H}^{(2)}}{D_{L H}^{(1)}+D_{L H}^{(2)}} \approx \frac{N_{H} m_{H}^{2}}{N_{L} m_{L}^{2}} \frac{4}{3+m_{H} / m_{L}},
$$

where $N_{L}$ and $N_{H}$ are the total number of light and heavy stars, $m_{L}$ and $m_{H}$ are the corresponding individual masses. $\Delta$ provides a measure of the importance of the heavy

$\S$ We note that 12 years before the work of BW, Gurevich (1964) derived a similar solution for how electrons distribute around a positively charged Coulomb center, which is the equivalent of the MBH in our case.

\|In this work, a nucleus is said to be a core if $\gamma<1$; it is said to be a cusp if $\gamma>1$. 
star's self-coupling relative to the light-heavy coupling (in terms of the $1^{\text {st }}$ and $2^{\text {nd }}$ order diffusion coefficients); and it depends essentially on the mass and number ratios, which is one parameter more than proposed by BW77. The weak branch, for $\Delta>1$ corresponds to the scaling relations found by BW77; while the strong branch, for $\Delta<1$, generalizes

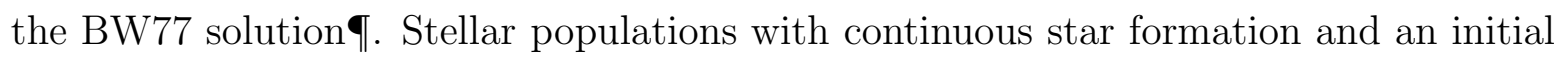
mass function (IMF) given by $d N / d M \propto M^{-\alpha}$ will be characterized by $\Delta<1$ if $\alpha \gtrsim 1.8$ and $\Delta<1$ otherwise; and, in particular, Salpeter and Kroupa's IMF generate evolved stellar populations with $\Delta<1$ (Alexander and Hopman 2009).

Figure 1. Evolution of density profiles. Mass density profiles, $\rho_{L}(r)$ (left panels) and $\rho_{H}(r)$ (right panels) at the end of the integrations, after $\approx 0.2 T_{\text {rlx }}\left(r_{\mathrm{h}}\right)$. Red curves are from FP calculations, green

curves are from NB simulations. The agreement between both methods is quite good. The mass ratio between heavy and light stars is $R=$ 10 , representing the expected typical mass ratio between light stars (MS stars, WDs and NSs) and stellar bhs, as explained in the text; the number fraction of heavy stars $f_{H}=2.5 \times 10^{-3}$ (top and middle panels) and $f_{H}=0.429$ (lower panels), corresponding to the strong and weak segregation regimes respectively. The initial condition is a Dehnen profile with central slope $\gamma=1$ for the top and bottom panels (Preto and Amaro-Seoane 2010a), $\gamma=1 / 2$ in the middle panel; a central $\mathrm{MBH}$ with $5 \%$ of the total mass of the cluster and $1 \%$ likewise. The particle number is $N=124,000$ (top and bottom) and $N=512,000$ (middle). The

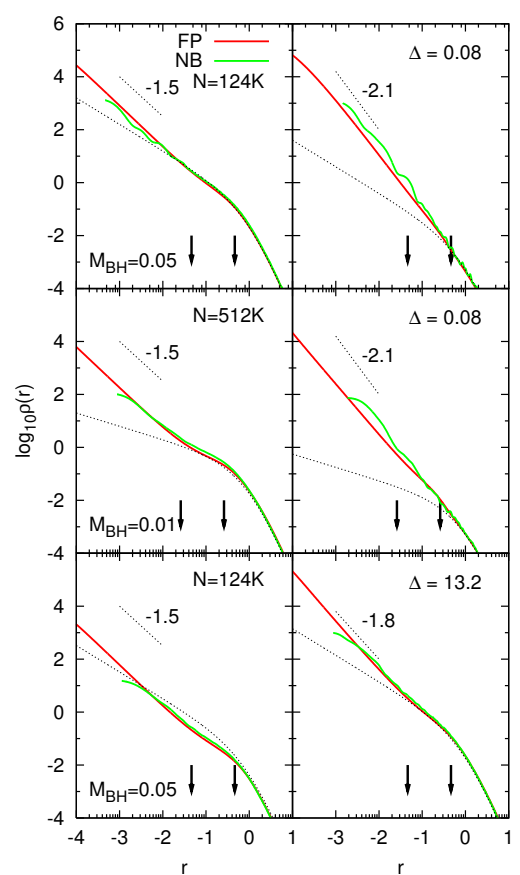
asymptotic slope $\gamma_{H}$ decreases from $\gtrsim 2$ to $\approx 7 / 4$ when moving from the strong to the weak branch of the solution. The asymptotic slope $\gamma_{H} \approx 3 / 2$ throughout, or just slightly below this value. The arrows point to radii $r_{h}$ and $0.1 r_{h}$.

There is a straightforward physical interpretation for the strong branch of mass segregation. In the limit where heavy stars are very scarce, they barely interact with each other and instead sink to the center due to dynamical friction against the sea of light stars. Therefore, a quasi-steady state develops in which the heavy star's current is not nearly zero and thus the BW77 solution does not hold exactly anymore. Indeed, in the limit where the number fraction $f_{H}$ of heavy stars is vanishingly small, as is the case of nuclei with realistic IMFs, the stellar potential is dominated by the light component. In this case, the light stars should evolve as if in isolation and develop a $\gamma_{L} \sim 7 / 4$ density cusp. The scarce heavy stars sink to the center due to dynamical friction against the

I The choice of the names is based upon the resulting slopes in the density profiles, which are steeper (stronger) or shallower (weaker) 
background of light stars, and will not exert any significant back-reaction on them (Preto 2010).

Figure 1 displays the FP and NB evolutions of the spatial density $\rho_{L}(r)$ and $\rho_{H}(r)$ for models with two mass components corresponding to different initial profiles, $\mathrm{MBH}$ masses and total particle number $N$. The starting models are either $\gamma=1$ or $\gamma=1 / 2$ Dehnen profiles for both components with a $\mathrm{MBH}$ of $1 \%$ or $5 \%$ of the total mass of the cluster. Dehnen density profiles are defined by $\rho(r)=(3-\gamma) M_{T O T} / 4 \pi r^{\gamma}\left(r_{b}+r\right)^{4-\gamma}$, have total stellar mass $M_{T O T}$, an inner (outer) logarithmic slope $\gamma(4-\gamma)$ and a break radius $r_{b}=1$ (which is larger than $r_{h}$ in all cases). We adopt units where $G=M_{T O T}=1$. The density of both components reaches a quasi-steady state within $\sim 0.2 T_{\mathrm{rlx}}\left(r_{h}\right)$, where $T_{\text {rlx }}\left(r_{h}\right)$ relaxation time measured at the influence radius (Preto and Amaro-Seoane 2010a). The top and middle panels display the strong mass segregation solution with $\gamma_{H} \sim 2.1$ as expected for $\Delta=0.08\left(f_{H}=2.5 \times 10^{-3}\right)$; while, in the bottom panel, $\Delta=13.2\left(f_{H}=0.429\right)$ displays the weak solution for which $\gamma_{H} \sim 7 / 4$. The former value was chosen to be close to the number fraction of stellar bhs to be expected from a standard Salpeter or Kroupa's IMF; the latter value is chosen to be representative of the regime of weak segregation studied by BW77. One can see from Figure 1 that in the case of weak segregation $\rho_{H}>\rho_{L}$ everywhere due to the extremely high number of heavy objects; in contrast, in the SMS regime $\rho_{H}>\rho_{L}$ only for $r \lesssim 0.01 r_{h}$ (and the light objects dominate in number almost everywhere). In all cases the asymptotic slopes are valid within $\sim 0.1 r_{h}$ and are totally predictable once $\Delta$ is known. These results agree pretty well with the predictions for the SMS regime (Alexander and Hopman 2009).

The particle number in our direct-summation $N$-body simulations sample ranges from $N=124,000$ to $N=512$, 000; our results do not show evidence of any dependence on total $N$, nor on the initial value of $\gamma$, once the results are re-scaled appropriately (i.e. measured in terms of the relaxation time). The agreement between NB and FP methods is quite good in all cases.

\section{Cusp Re-growth}

\subsection{Current observations: A missing cusp}

We have seen that theory predicts a steady state cusp that reaches extremely high densities in the center near the $\mathrm{MBH}$. Furthermore, given a normalization at, say, $r_{h}$ and a knowledge of the stellar mass function (and thus of $\Delta$ ), the density profile inside $r_{h}$ becomes completely determined. But observations are much more complicated to interpret. First, one must realize that there are very few galaxies for which the influence radius $r_{h}$ can be resolved. In fact, except for the nearest galaxies, $r_{h}$ covers an angular region in the sky which is too small to be resolved even with the HST. The HST has an angular resolution of $\sim 0.1^{\prime \prime}$. In the case of galaxies in the Virgo cluster, at $\sim 17 \mathrm{Mpc}$ of distance, it can only resolve regions with linear sizes $\gtrsim 8.25$ pc. Therefore, HST would not be able to resolve SgrA*'s radius of influence if it were at the distance of Virgo. 
Since $r_{h} \propto M_{\bullet}^{1 / 2}$, it can only start to resolve the influence radius of Virgo's MBHs that have masses $M_{\bullet} \gtrsim 4 \times 10^{7} M_{\odot}$. Second, even in the few cases for which $r_{h}$ can be resolved to some extent, it still is necessary to assess whether the observed stars (only those that are bright enough to be detected) really trace the underlying (dynamically dominant) invisible population. Third, given the fact that, as we have seen, stars tend to segregate by mass, there is an extra uncertainty related to the unknown stellar mass function. Moreover, there are indications that star formation events are common in galactic nuclei and furthermore that the resulting IMF in these sub-parsec regions may be substantially different from that of the field stars and biased towards heavy masses (Bartko et al. 2010). Finally, it is necessary to deproject the observations and, in the (inevitable) absence of complete knowledge of phase space coordinates, one must rely on kinematic assumptions regarding the (an-)isotropy of stellar velocities and on the three dimensional shape of the stellar system.

Figure 2. Time for cusp re-growth. Single-mass relaxation time at $r_{h}$ for singlemass cored models as a function of MBH mass. The shaded area covers $\left[0.1 T_{\mathrm{rlx}}, 0.2 T_{\mathrm{rlx}}\right]$ - the time for cusp regrowth if there is no hole in the initial DF. The three dashed lines above the shaded region represent the average time needed for the cusp re-growth in case one imposes an initial cavity with size $R_{\text {cav }}=0.5,1$ or $2 \mathrm{pc}$. The horizontal dashed curve represents 13 Gyr. It can be seen that the time needed to re-grow a cusp around $\mathrm{MBH}$ with masses $M_{\bullet} \lesssim 5 \times 10^{6} M_{\odot}$

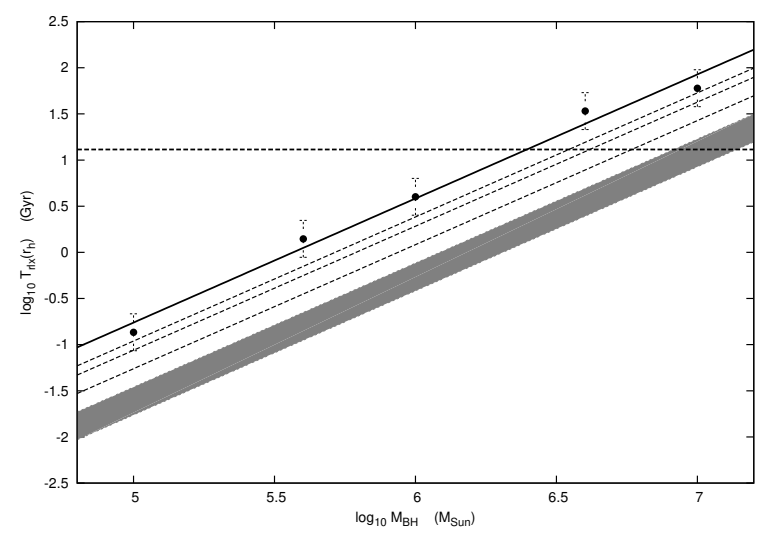
is below a Hubble time so long as the initial cavity is smaller than $\lesssim 2$ pc.

Nevertheless, it has come as a surprise that very recent spectroscopic observations of the Galactic center revealed a core (or even a dip) in the surface distribution of the old stellar population (essentially red giants) which should have had time to relax into a cuspy density profile (Buchholz et al. 2009; Do et al. 2009). The caveat is that the detected stars are still a small fraction, of about $5 \%$, of the stellar population as a whole and therefore do not exclude the presence of an extended dark cluster (presumably made of stellar bhs and other CRs) - which would indeed agree with our theoretical expectations.

\subsection{Carving a hole in the stellar distribution}

To assess the likelihood that the Galactic center is indeed unrelaxed, it is natural to ask: how long does it take to re-grow a cusp if, at some point, it has been destroyed? A complete answer will, of course, depend on the extent to which the cusp was destroyed, i.e. how much mass was expelled from the original cusp and over which radial range. 
At this level, it does not matter very much which mechanism led to the destruction of the cusp. We discuss briefly possible scenarios for cusp destruction at the end of the section.

In order to investigate this question, we have concocted a set of initial conditions purported to mimic the outcome of a destroying cusp event-such as the carving of a cavity in phase space through the ejection of stars by, say, an infalling IMBH or, following a major merger, by a $\mathrm{MBH}$. We model the outcome of such an event by imposing that all stars with binding energies larger than some $E_{0}$ or almost equivalently, with semimajor axis smaller than $G M_{\bullet} / 2 E_{0}$, are not present in the initial DF. In fact, inside $r_{h}$ the MBH dominates the gravitational potential and $E \sim G M_{\bullet} / 2 a$. We thus set up an initial Dehnen model with $f(E)=0$ for $E>E_{0}$-in other words, there is an initial cavity in the phase space DF, but not in physical space as the stars with lower energy still entail $\rho(r) \sim r^{-1 / 2}$ at the center, although with a smaller amplitude than the original model. The values of $E_{0}$ were chosen to represent cavities of size $R_{c}=0.5,1,2$ pc resulting from the inspiral of an IMBH/MBH. Note that these models are, by construction, isotropic in the velocity distribution. + Our fiducial model is a Milky Way type nucleus with $M_{\bullet}=4 \times 10^{6} M_{\odot}$, some $10^{6} M_{\odot}$ in total stellar mass inside $1 \mathrm{pc}$ distributed according to an initial central density slope $\gamma=1 / 2$, two components with masses $m_{L}=1 M_{\odot}$ and $m_{H}=10 M_{\odot}$, and $0.1 \%$ of stellar bhs by number. When the stellar distribution has no phase space cavity, this translates into having stars down to roughly $10^{-5}$ pc. Having validated the FP models with detailed $N$-body simulations, we now proceed in the rest of the paper to describe results obtained with the (much faster) FP approach.

Figure 2 shows the times for cusp re-growth computed with FP for different galactic nuclei models. The time for cusp re-growth is defined as the time it takes for a given initial density profile $(\rho(r)$ in space or $f(E)$ in phase space, with or without an initial cavity) to reach its asymptotic slope, which depends on $\Delta$, down to $r \sim 0.01 r_{h}$. This is indeed the scale which is resolved by recent observations of the Galactic center (Schödel et al. 2009). The shaded region represents the time of cusp re-growth for a range of $R$ and $f_{H}$ (all in the SMS regime, $\Delta<1$ ) for the case where $f(E)$ extends to high $E$ without any cut. It can be seen that, for $M_{\bullet} \lesssim 10^{7} M_{\odot}$, cusps grow in less than a Hubble time; in the particular case of the Milky Way nucleus with $M_{\bullet} \sim 4 \times 10^{6} M_{\odot}$, it takes no longer than $\sim 4.8$ Gyr to fully re-grow a steady-state, mass segregated, stellar cusp and only $\sim 2.4$ Gyr to have it grown down to $0.01 r_{h}$. If, instead, an initial cavity is imposed at the center with size $R_{\text {cav }}=0.5,1$ or 2 pc in case of the Milky Way (or $R_{\text {cav }}=0.2 r_{h}, 0.4 r_{h}$ or $0.8 r_{h}$ in case of a generic nucleus), times for re-growth are represented by the dashed curves above the shaded region. In this case, times for cusp re-growth increase; in the Milky Way case, it becomes $\sim 4.8,7.2$ or 12 Gyr, respectively. Note that, in the mass range $10^{5}-10^{6} M_{\odot}$, the times for cusp re-growth are definitely much shorter than a Hubble time - even if a fairly large cavity of size comparablee to $r_{h}$

+ We assume that the timescale for isotropization of velocities is much shorter than that associated with the cusp re-growth; in any event this should not affect our estimates by more than $10 \%$ or $20 \%$ maximum. 
is hypothesized. The full curve represents the relaxation times computed at the radius of influence $r_{h}$, while the dashed curves represent the actual times for cusp re-growth as measured from FP calculations (Preto and Amaro-Seoane 2010a).

It is difficult to devise plausible mechanisms for the formation of such large cores in the stellar distribution. For instance, the inspiral of an IMBH of mass $M_{\bullet} \sim 10^{3-4} M_{\odot}$ that forms an unequal-mass binary with the $\mathrm{MBH}$ and ejects stars through three body encounters would tend to progressively wipe out the stellar cusp. However, the core radius carved by such an event is $r_{c} \sim 0.02-0.04$ pc (Baumgardt et al. 2006) and thus a steady inflow of such IMBHs (one every $10^{7}$ years for a Hubble time) would be required in order to carve a large core 50 or 100 larger. Such large inflow of IMBHs have been proposed by Portegies Zwart et al. (2006). This does not seem very likely anymore in light of the fact that such IMBHs were hypothesized to be formed by runaway mergers of stars in the center of globular clusters. However, at solar metallicities, such mechanism seems very inneficient. Mass loss due to very strong winds severely limits the growth of the stellar object being formed and the likely end result of a runaway merger is a $\sim 100 M_{\odot}$ Wolf-Rayet star. At lower metallicities, mass loss is lower and the remnant can be more massive $\sim 260 M_{\odot}$, but in any case it will not form an IMBH (Glebbek et al. 2009). In sum, it looks very unlikely that sufficient IMBHs can be formed in order to generate such steady inflow to the Galactic center. Another possibility would be that $\mathrm{SgrA}^{*}$ is a binary $\mathrm{MBH}$, but this would most likely imply that there has been a, more or less recent, major merger involving the Milky Way. This would contradict the apparent pure-disk nature of the Galaxy, as theoretical interpretations of stellar kinematic data of the Galactic Bulge seem to favor that the Bulge is part of the disk and not a separate component resulting from a merger Shen et al. (2010)y - aside from the fact that there are strong constraints from the SgrA* proper motion (Reid and Brunthaler 2004).

Stars in a Keplerian potential, $G M_{\bullet} / r$, do not precess because of the $1: 1$ resonance between their radial and azimuthal frequencies. Resonant relaxation (RR) results from the coherent torques that such stars exert on each other leading to a fast evolution of their orbital angular momenta over timescales $\lesssim T_{p r}$, where $T_{p r}$ is the precession timescale due to departures from an exact Kepler potential (Rauch and Tremaine 1996). Madigan et al. (2010) suggest that RR, by increasing the rate of angular momentum diffusion in the near-Keplerian gravitational potential around the $\mathrm{MBH}$, may boost the tidal disruption rate of stars and could thus create a near-cavity (out to $\sim 0.1 \mathrm{pc}$ ) in the stellar distribution. It is certain that $\mathrm{RR}$ operates to some extent in the inner parsec, but we doubt it can completetly explain the dearth of red giant stars there or, more generally, the full destruction of a cusp - including CRs such as stellar bh: First, their final density distribution does not show a cavity, nor a shallow cusp profile, for $r \gtrsim 0.1 \mathrm{pc}$; instead, they get final slopes $\gamma \sim 1.5$ for $r \gtrsim 0.1 \mathrm{pc}$. This is in contrast with observations which show a decaying density for $r \lesssim 0.24$ pc (Buchholz et al. 2009). Second, we believe that their Monte-Carlo calculations severely underestimate

* Resonant relaxation is, nevertheless, very likely to have a significant impact on the EMRI event rates (Hopman and Alexander 2006). 
the rate at which the cusp re-grows; in fact, they obtain a timescale which is $\sim 10-30$ times longer than that obtained with our $N$-body simulations (which are free from any simplifying dynamical assumptions), and also from ours and their own recent FP calculations (Hopman and Madigan 2010). Third, were they to use the latter rates, and given that the time $R R$ takes to deplete the cusp is of the same order as the time we obtain for cusp re-growth, the net effect of RR on the cusp would likely be minute. Moreover, stellar bhs cannot be tidally disrupted, and this makes them less susceptible to be extracted from the cusp than $10 M_{\odot}$ stars.

\section{EMRIs rates}

\subsection{Adiabatic and abrupt EMRIs: Estimation of the rates}

Given a steady state stellar bhs continue to diffuse in $(E, J)$-space and some of them eventually come into close interaction with the MBH. During a close interaction, a stellar bh can either be promptly scattered into the $\mathrm{MBH}$, accompanied by a single or a few brief bursts of GWs in the LISA band - the so-called "direct-plunges" —, though they are not likely detectable unless if emitted from the Galactic center (Hopman et al. 2007), or scattered outwards in the cusp. In either case, it does not live enough to become an EMRI. Alternatively, it may undergo a very slow, adiabatic, inspiral without being appreciably disturbed by other stars and, in this case, it will eventually become an EMRI detectable by LISA. An EMRI object thus has to spend very many orbits without being significantly scattered by the gravitational tugs of the other stars. In other words, they must fullfill the following inspiralling criterion: the time $T_{\mathrm{GW}}$ it takes for the inspiral, due to orbital energy lost by GW emission only, must be shorter than the typical time $T_{J}$ it takes on average to drift in angular momentum by an amount $J$ which equals its orbital angular momentum. Otherwise, they will be promptly captured by the MBH before entering the LISA band. The inspiral criterion can be stated in terms of the parameter $s$ being smaller than unity, $s=T_{\mathrm{GW}} / T_{J}<1$. For $T_{\mathrm{GW}}>T_{J}$, it is almost certain that this object has either taken an almost radial orbit and fallen into the $\mathrm{MBH}$ as a direct plunge or has been scattered outwards. $甘$ It turns out that this parameter simply scales with orbital's semimajor axis: $s \propto a^{3 / 2-p}$ (Hopman and Alexander 2005), which means that it is a decreasing function of $a$ so long as $p<3 / 2$. This is indeed the case in both regimes of mass segregation. Furthermore, Hopman and Alexander (2005) have shown that the probability for a successful inspiral as a function of orbital semimajor axis (or energy) is almost a step function of semimajor axis. If $a<a_{\mathrm{GW}}$, it is almost certain that the stellar bh will become an EMRI; it will almost certainly not become one in case the inequality sign is reversed (and the width of the "transition region" is very small). This crucial threshold quantity demarcates the orbits which are close enough to the MBH to sucessfully decouple from the rest of the cluster and undergo

\# In steady state, on average each star that drifts outward by an amount $J$ will be compensated by another that drifts inward by the same amount. This balance only breaks down for those orbits that fall on to the hole, since there are obviously no stars coming out of it to keep detailed balancing. 
the slow, adiabatic inspiral that defines an EMRI from those more weakly bound orbits that will be perturbed out of the EMRI tracks due to scattering with other stars.

Therefore, in order to estimate the EMRI event rate, given a steady state $f(E)$, obtained via the FP equation, one essentially counts the number of stars that populate the region of phase space for which the inspiralling criterion above is satisfied and divide it by the local relaxation time. Note that here, for simplicity, we ignore other driving mechanisms - in particular, we ignore resonant relaxation. Under these assumptions, the EMRI rate for stellar bhs is approximately given by

$$
\Gamma_{\mathrm{EMRI}}=f_{\bullet} \int_{E_{\mathrm{GW}}}^{+\infty} d E \frac{n(E)}{\ln \left(J_{c}(E) / J_{\mathrm{lc}}\right) T_{\mathrm{rlx}}(E)},
$$

where $f_{\bullet}$ is the number fraction of SBHs in the stellar population, $n(E)$ is the total number of stars per unit energy $(n(E) \propto f(E)$, see Preto $(2010)), J_{c}(E)=\sqrt{G M_{\bullet}} / 2 E$ is the specific angular momentum of a circular orbit of energy $E, J_{\mathrm{lc}}=4 G M_{\bullet} / c$ is the losscone angular momentum and $T_{\mathrm{rlx}}=0.34 \sigma^{3} /\left[G^{2}\left(m_{\bullet} \rho_{\bullet}+m_{*} \rho_{*}\right) \ln \Lambda\right]$ is the relaxation time. The log term in the denominator in (2) arises from the phase space (partial) depletion resulting from the presence of the loss cone. The conversion between $r$ and $E$ is, for $r \ll r_{h},\langle E(r)\rangle=G M_{\bullet} / 2 r$ or $E=G M_{\bullet} / 2 a$. The critical radius $a_{\mathrm{GW}}$, or energy $E_{\mathrm{GW}}$, for EMRIs is approximately $a_{\mathrm{GW}}=0.01 r_{h}$; and, to first order, $a_{\mathrm{GW}}$ is independent of $M_{\bullet}$ (Hopman and Alexander 2005).

\subsection{The relevance of realistic models of mass segregation for the rates}

The weak regime of SMS, and corresponding BW solution, would lead to a fairly high intrinsic rate, per galaxy, of EMRIs. In fact, Figure 3 shows that, for a Milky Way nucleus, in case $\Delta>1$, the intrinsic EMRI rate is $\gtrsim 10^{3}$ per Gyr. This is, however, unrealistic as such scenario pressuposes an unrealistically high number fraction of bhs $(f \bullet \gtrsim 0.0325$ for $\Delta>1)$. In the more realistic case, when $\Delta \sim 0.03$ the BW solution would entail a strong supression of the EMRI rate to - at best - a few tens of events per Gyr. This is where SMS solution appears to rescue us. SMS implies a higher density of bhs inside $r_{h}$ as compared with the $\gamma=7 / 4$ solution, and in this way-by decreasing the local $T_{r l x}$ and increasing $n(E)$ close to the $\mathrm{MBH}$ - it partially, but not completely, compensates for the small number fraction of bhs entailed by realistic mass functions.

Figure 3. EMRI rate as a function of $\Delta$. The number of stellar bh EMRI events per Gyr in a Milky Way type nucleus $\left(M_{\bullet}=4 \times 10^{6} M_{\odot}\right.$ and $\left.M_{*}(<1 \mathrm{pc})=10^{6} \mathrm{M}_{\odot}\right)$ 
as a function of the parameter $\Delta$. This is computed from a two-component mass segregated stellar cusp $\left(\gamma_{H} \approx 2.1\right.$ and $\left.\gamma_{L} \approx 1.5\right)$ with mass ratios $R=$ 10 and 15 obtained from FP calculations. In the case of the fiducial value $f_{\bullet}=10^{-3}, \Delta \approx$ 0.03 ; in those circumstances, each Milky Way like nucleus will produce on average $\sim 250$ stellar bh EMRIs per Gyr.

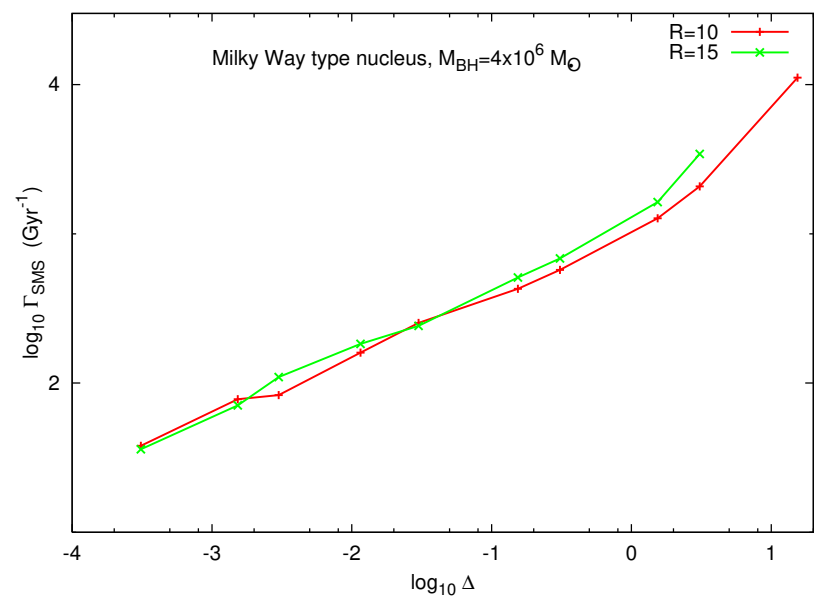

In order to quantitatively evaluate the boost $\Gamma_{\mathrm{SMS}} / \Gamma_{\mathrm{BW}}$ to the EMRI rates from SMS, for a given $\Delta$ and a fixed mass normalization at $r_{h}$, one needs to estimate what would be the rate if the spatial and phase space densities were determined by the $\gamma=7 / 4$ cusp for $r \lesssim 0.1 r_{h}$. This is done as follows: we define analytically both $\rho(r)$ and $f(E)$ that would result from a $\gamma=7 / 4$ inside $0.1 r_{h}$

$$
\begin{aligned}
& \rho(r)=\rho_{F P}(r), \quad r>r_{L} \\
& \rho(r)=\rho_{F P}\left(r_{L}\right) \times\left(\frac{r_{L}}{r}\right)^{7 / 4}, \quad r \leq r_{L},
\end{aligned}
$$

and

$$
\begin{aligned}
& f(E)=f_{F P}(E), \quad E<E_{L} \\
& f(E)=f_{F P}\left(E_{L}\right) \times\left(\frac{E}{E_{L}}\right)^{1 / 4}, \quad E \geq E_{L},
\end{aligned}
$$

where the indices FP mean that the profile is taken from the Fokker-Planck calculation. $r_{L}$ (and $E_{L}$ ) is a reference radius (energy) chosen according to $r_{L} \sim 0.1 r_{h}$.

Figure 4. Boost on the EMRI rates from strong segregation.

One can see that, for values of $\Delta<1$, there is a significant boost to the EMRI rates in comparison to which it would result in the case of a $\gamma=7 / 4$ BW cusp. In particular, for our fiducial value $\Delta \sim 0.03\left(f_{\bullet} \sim\right.$ $\left.10^{-3}\right)$, the boost is of order of a factor 10 with respect to a $7 / 4$ BW cusp with the same mass normalization at $r=1 \mathrm{pc}$.

Figure 4 shows the boost to

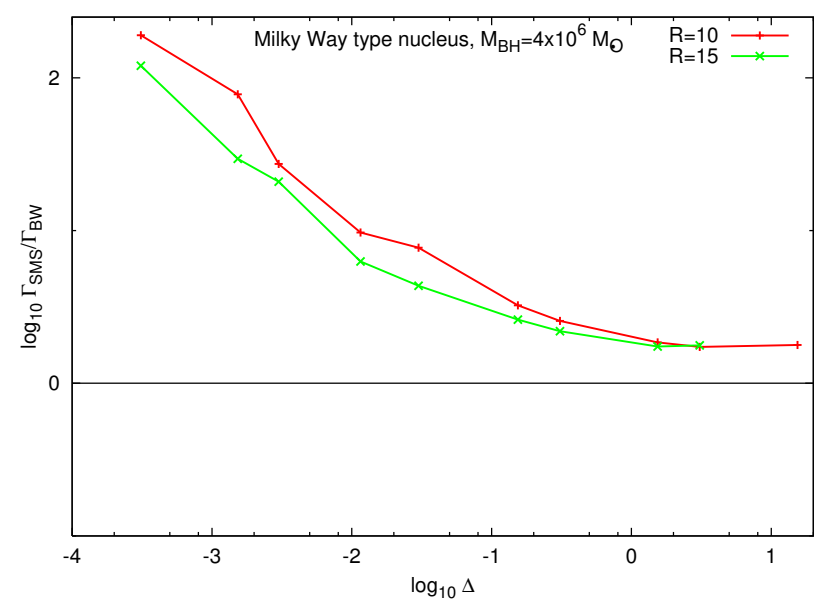
the EMRI rates due to SMS relative to what would be obtained from a BW profile. 
Going from an unrealistically high $f_{\bullet}$, as adopted by BW77 (say $\Delta=3$ ), to a more realistic $f_{\bullet}$ (say $\Delta=0.03$ ), while neglecting the existence of SMS, one supresses the EMRI rate by factors of $\sim 100-150$ (the former would lead to $\sim$ few $\times 10^{3}$ EMRIs per Gyr; the latter is reduced to $\sim$ few tens per Gyr). However, by taking into account the SMS solution, for this low $\Delta=0.03$, we boost again the rates by a factor close to 10 , thus partially compensating the reduction of EMRIs (from few tens to a few hundred per Gyr; in fact, there are $\sim 250$ per Gyr in case $\Delta=0.03$ for a Milky Way nucleus). We conclude that the apparently inocuous and tiny change of the logarithmic slope from $\gamma_{H}=7 / 4$ to $\gamma_{H} \sim 2$ can have a substantial effect (a factor of $\sim 10$ ) on the expected EMRI rate.

Figure 5. EMRI rates as a function of MBH mass in strongly segregated nuclei. The EMRI rate depends on the MBH mass, $\Gamma_{S M S} \propto M_{\bullet}^{-0.19}$. Shown are curves for $\Delta=0.03$ $\left(f_{\bullet}=10^{-3}\right.$ and $\left.10^{-2}\right)$ and three different mass ratios between heavy and light stars, $R=10$ and 15 .

Figure 5 shows the dependence of the intrinsic EMRI rate on the mass of the central $\mathrm{MBH}$, where the validity of the $M_{\bullet}-\sigma$ relation was assumed

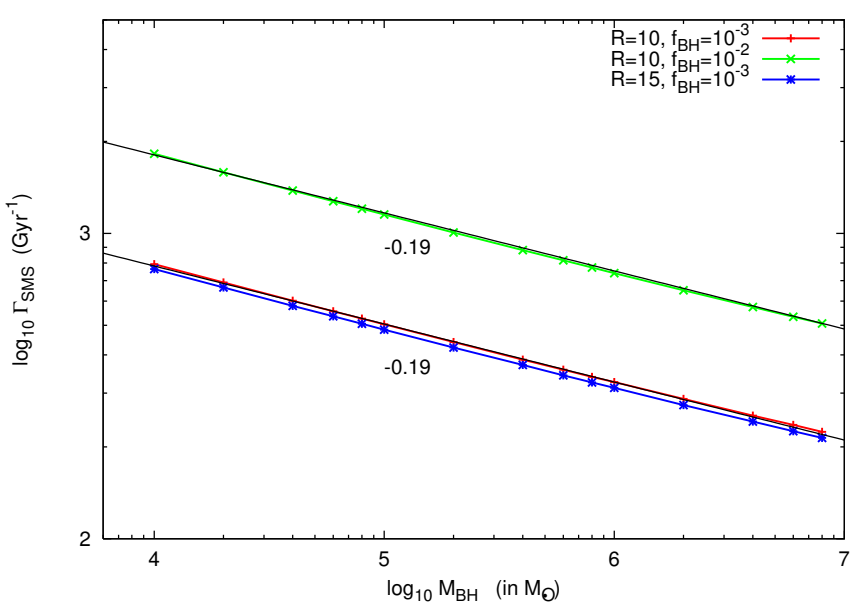
(Ferrarese \& Ford 2005). We see that the EMRI rate for stellar bhs scales as $\propto M_{\bullet}^{-0.19}$, independently of $R$ and $f_{\bullet}$. Its absolute normalization depends obviously on the number fraction $f_{\bullet}$ of sbhs, in agreement with Figures 3 and 4.

One can make a rough conversion of the estimated intrinsic rate into LISA detection rates. Following Gair (2009), who made a number of assumptions regarding the local density of MBHs and its spin distribution, plus on the LISA detection capabilities, we find that according to its equation (7), LISA will see around $\sim 10^{2}-7 \times 10^{2}$ EMRI events during a 2-year or 5-year mission, respectively. Note that these rates may change by factors of $\sim 2-3$ as a function of corrections to the local MBH mass density (Graham and Driver 2007); moreover, the uncertainties regarding the efficiency of RR and other channels may still affect the rate predictions by one or two orders of magnitude. A lot of work still remains to be done; nevertheless, the consequences regarding the SMS regime are significant and under control.

\section{Conclusions}

We have considered simplified stellar models of galactic nuclei, with only two mass components, which harbor MBHs that fall into the LISA detection bandwidth. For quite generic initial conditions, such stellar clusters are expected to have reached a relaxed, 
mass segregated, steady state which is independent of initial conditions at the time of formation. Strong (realistic) mass segregation is a robust outcome from the growth and evolution of stellar cusps around MBHs in the mass range $10^{4}-10^{7} M_{\odot}$ to which LISA will be sensitive. Our N-body results validate the Fokker-Planck description of the bulk properties of the stellar distribution. SMS boosts the EMRI event rates with respect to what would be implied by a shallower stellar density profile (e.g. $\gamma=7 / 4$, which has been the working assumption of almost all event rate estimates in the literature so far) that also respect the mass normalization obtained from observations of the Galactic center at $1 \mathrm{pc}$ from the hole. In particular, our fiducial models of the Galactic center are enhanced by a factor of $\sim 10$-leading to a predicted value of $\sim 250$ stellar bh EMRIs per Gyr. The FP formalism assumes two-body relaxation as the only dynamical driver present - this could be a severe restriction at radii $\lesssim 0.01 r_{h}$, inside which even the NB simulations with higher $N$ in our sample start to run out of particles and where RR could play an important role (Hopman and Alexander 2006; Madigan et al. 2010). Other crucial mechanisms are resonant relaxation, (small) triaxiality of the galactic potential, tidal separation of binaries and massive perturbers (see e.g. Amaro-Seoane et al. 2007, for a review). These are the subject of our current research work, and the extent to which they can significantly affect the EMRI rates is still an open question.

\section{Acknowledgments}

It is a pleasure for PAS to thank Tom Prince for the invitation to give a plenary talk in the symposium. He is equally indebted with Esmeralda Martínez for discussions as well as with Vivian J. Drew for her great sense of humour and coordination of the workshop. MP thanks Lauren B. for interesting comments. PAS and MP are partially supported by DLR (Deustches Zentrum für Luft- und Raumfahrt). The simulations have been carried out on the dedicated high-performance GRAPE-6A clusters at the Astronomisches Rechen-Institut in Heidelberg tt, which was funded by project I/80 041043 of the Volkswagen Foundation and by the Ministry of Science, Research and the Arts of Baden-Württemberg (Az: 823.219-439/30 and /36), an also at the TufFstein cluster of the AEI.

\section{References}

Alexander T and Hopman C 2003 ApJ Lett. 590, 29-32.

Alexander T and Hopman C 2009 ApJ 697, 1861-1869.

Amaro-Seoane P, Gair J R, Freitag M, Miller M C, Mandel I, Cutler C J and Babak S 2007 Classical and Quantum Gravity 24, 113-+.

Babak S, Baker J G, Benacquista M J, Cornish N J, Larson S L, Mandel I, McWilliams S T, Petiteau A, Porter E K, Robinson E L, Vallisneri M, Vecchio A, Data Challenge

†† GRACE: see http://www.ari.uni-heidelberg.de/grace 
Task Force t M L, Adams M, Arnaud K A, Błaut A, Bridges M, Cohen M, Cutler C, Feroz F, Gair J R, Graff P, Hobson M, Shapiro Key J, Królak A, Lasenby A, Prix R, Shang Y, Trias M, Veitch J, Whelan J T and participants 2010 Classical and Quantum Gravity 27(8), 084009-+.

Bahcall J and Wolf R 1976 ApJ 209, 214-232.

Bahcall J and Wolf R 1977 ApJ 216, 883-907 (BW77).

Barack L and Cutler C. 2004 Phys Rev D 69, 082005.

Bartko, H.; Martins, F.; Trippe, S.; Fritz, T. K.; Genzel, R.; Ott, T.; Eisenhauer, F.; Gillessen, S.; Paumard, T.; Alexander, T.; Dodds-Eden, K.; Gerhard, O.; Levin, Y.; Mascetti, L.; Nayakshin, S.; Perets, H. B.; Perrin, G.; Pfuhl, O.; Reid, M. J.; Rouan, D.; Zilka, M.; Sternberg, A. 2010 ApJ 708, 34-840

Baumgardt H, Makino J and Ebisuzaki T 2004 ApJ 613, 1133-1142.

Baumgardt H, Gualandris A and Portegies Zwart S T 2006 MNRAS 372, 174-182.

Binney, J. andTremaine, S. Galactic Dynamics (Princeton, US: Princeton University Press, 2008.)

Buchholz R., Schödel, R. and Eckart, A. 2009 A\& A, 499, 483

Di Matteo, T., Colberg, J., Springel, V., Hernquist, L. andSijacki, D. 2008 ApJ 676, 33.

Do, T., Ghez, A. M., Morris, M. R., Lu, J. R., Matthews, Keith andYelda, S., andLarkin 2009 ApJ 703, 1323.

Eisenhauer F, Genzel R, Alexander T, Abuter R, Paumard T, Ott T, Gilbert A, Gillessen S, Horrobin M, Trippe S, Bonnet H, Dumas C, Hubin N, Kaufer A, Kissler-Patig M, Monnet G, Ströbele S, Szeifert T, Eckart A, Schödel R and Zucker S 2005 ApJ 628, 246-259.

Ferrarese, L. \& Ford, H. 2005, Space Sci. Rev., 116, 523

Freitag M 2003 ApJ Lett. 583, 21-24.

Gair J 2009 Classical and Quantum Gravity 26, 094034.

Ghez A M, Salim S, Hornstein S D, Tanner A, Lu J R, Morris M, Becklin E E and Duchêne G 2005 ApJ 620, 744-757.

Ghez A M, Salim S, Weinberg N N, Lu J R, Do T, Dunn J K, Matthews K, Morris M R, Yelda S, Becklin E E, Kremenek T, Milosavljevic M and Naiman J 2008 ApJ 689, 1044-1062.

Gillessen S, Eisenhauer F, Trippe S, Alexander T, Genzel R, Martins F and Ott T 2009 ApJ 692, 1075-1109.

Glebbeek, E.; Gaburov, E.; de Mink, S. E.; Pols, O. R.; Portegies Zwart, S. F. 2009 A\&A 497, 255-264

Graham A.W. and Driver S.P. 2007 MNRAS 380, L15-L19.

Gurevich A. 1964, Geomag. Aeronom., 4, 247

Hopman C and Alexander T 2005 ApJ 629, 362-372. 
Hopman C and Alexander T 2006 ApJ 645, 1152-1163.

Hopman C and Alexander T 2006 ApJL 645, 133-136.

Hopman C and Freitag M and Larson S L 2007 MNRAS 378, 129-136.

Hopman C and Madigan A.-M. 2010 arXiv:1002.1220.

Khalisi E, Amaro-Seoane P and Spurzem R 2007 MNRAS 374, 703-720.

Kroupa P 2001 MNRAS 322, 231-246.

Lightman A and Shapiro S 1977 ApJ 211, 244-262.

Madigan A.-M., Hopman, C. and Levin Y. 2010 arXiv:1010.1535.

Merritt D 2010 ApJ 718, 739-761.

Preto M 2010 GW Notes 3, 3-27.

Preto M, Merritt D and Spurzem R 2004 ApJL 613, 109-113.

Preto M and Amaro-Seoane P 2010 ApJL 708, 42-46.

Preto M and Amaro-Seoane P 2011, to be submitted to ApJ

Portegies Zwart, Simon F.; Baumgardt, Holger; McMillan, Stephen L. W.; Makino, Junichiro; Hut, Piet; Ebisuzaki, Toshi 2006 ApJ 641, 319-326

Rauch K and Tremaine S 1996 New Astronomy 1, 149-170.

Reid M J and Brunthaler A 2004, ApJ, 616, 872-884

Schödel R, Merritt D andEckart A 2009, A\& A, 502, 91

Shen, Juntai; Rich, R. Michael; Kormendy, John; Howard, Christian D.; De Propris, Roberto; Kunder, Andrea 2010 ApJL,720, L72-L76

Sigurdsson S and Rees M J 1997 MNRAS 284, 318-326.

Spitzer L 1987 Dynamical evolution of globular clusters Princeton, NJ, Princeton University Press, 1987, $191 \mathrm{p}$.

Volonteri, M. 2010, A\&A Rev , 18, 279 